\title{
Motor problems in children with neurofibromatosis type 1
}

André B. Rietman ${ }^{1,6^{*}}$, Rianne Oostenbrink ${ }^{2}$, Sanne Bongers ${ }^{1}$, Eddy Gaukema ${ }^{5}$, Sandra van Abeelen ${ }^{5}$, Jos G. Hendriksen ${ }^{5}$, Caspar W. N. Loomann ${ }^{4}$, Pieter F. A. de Nijs ${ }^{1}$ and Marie-Claire de Wit ${ }^{3}$

\begin{abstract}
Background: Children with the neurogenetic disorder neurofibromatosis type 1 (NF1) often have problems with learning and behaviour. In both parent reports and neuropsychological assessment, motor problems are reported in approximately one third to one half of the children with NF1. Studies using broad motor performance test batteries with relatively large groups of children with NF1 are limited. The aim of this cross-sectional observational study was to describe the severity of motor problems in children with NF1 and to explore the predictive value of demographics, intelligence, and behavioural problems.

Methods: From 2002 to 2014, 69 children with NF1, aged 4 to 16 years (age $=9.5 \pm 2.8$ years; 29 girls) had a motor, psychological, and neurological evaluation in an NF1 expertise centre. Data were collected about (1) motor performance (M-ABC: Movement Assessment Battery for Children), (2) intelligence, and (3) emotional and behavioural problems as rated by parents.

Results: Sixty-one percent of these children scored within the clinical range of the M-ABC. In ordinal logistic regression analyses, motor problems were associated with symptoms of attention-deficit/hyperactivity disorder (ADHD), symptoms of autism spectrum disorder (ASD), and externalising behavioural problems. Motor outcome was not predicted by age, intelligence, scoliosis, hypotonia, nor hypermobility.

Conclusions: Motor problems are among the most common comorbid developmental problems in children with NF1, and these problems do not diminish with age. Because of their impact on daily functioning, motor problems need to be specifically addressed in diagnosis, follow-up, and treatment of NF1.
\end{abstract}

Keywords: Neurofibromatosis type 1, Motor problems, DCD, Emotional and behavioural problems, Intelligence

\section{Background}

Neurofibromatosis type 1 (NF1) is an autosomal dominant neurogenetic disorder with an incidence of at least 1:2700 [1]. Although NF1 is defined by cutaneous and neurological symptoms such as café-au-lait spots and neurofibromas, the most common complications in childhood are deficits of cognition and of social and emotional development [2]. The prevalence of

\footnotetext{
*Correspondence: a.rietman@erasmusmc.nl

'Department of Child and Adolescent Psychiatry/Psychology, ENCORE NF1 Expertise Centre for Neurodevelopmental Disorders, Erasmus Medical Centre-Sophia Children's Hospital, Rotterdam, The Netherlands

${ }^{6}$ Department of Child and Adolescent Psychiatry/Psychology, Sophia Children's Hospital, Room Sp 2478, P.O. Box 20603000 CB Rotterdam, The Netherlands

Full list of author information is available at the end of the article
}

neuropsychiatric problems such as attention-deficit/ hyperactivity disorder (ADHD) and autism spectrum disorder (ASD) is much larger than in the general population [3]. In both parent reports and neuropsychological assessments, motor problems are reported in approximately one third to one half of the children with NF1 [4, 5]. Almost $30 \%$ of children with NF1 had received occupational therapy [6], and over $40 \%$ receive remedial teaching for motor problems at school [7]. NF1-related skeletal and muscular abnormalities, such as scoliosis, pseudo-arthrosis, decreased bone strength, and reduced muscle strength may be associated with motor problems in NF1 [5]. Motor problems can hinder a child's participation at school and in play, sports, and peer-group activities, but they may also affect social and emotional 
development [8]. In our expertise centres for NF1, motor problems are among the most common complaints, which is the reason for the structural assessment of motor skills presented in this study.

Previous studies on motor skills in NF1 have often used selective tests, targeting only parts of the motor domain $[2,9]$. Studies using a small selection of motor or constructional tests do not show the full range of motor problems in children with NF1. Broader test batteries for both fine and gross motor skills have been used in a limited number of smaller studies $[5,10]$ or when focusing on young children $[4,11]$. Recently, [9] a broad test battery (the BOT-2) was used with 46 children, from 7 to 17 years old, to establish correlations between problems in motor and cognitive domains. In this study, cognition was associated with balance, gait, running speed, and agility in children with NF1. A shared abnormal neurodevelopmental process underlying cognitive and motor abilities in NF1 was hypothesised [9].

A study on a large group of children and adolescents with NF1, using a broad test battery for motor performance, could inform health care professionals not only about the association between motor problems and cognitive development but also about the association with the emotional and behavioural problems often present in NF1. Our cross-sectional study aims to describe the presence and severity of motor problems in children and adolescents with NF1 and to explore the associations between these motor problems and background variables, intelligence, and emotional and behavioural problems.

\section{Methods}

\section{Procedure and patients}

The Kempenhaeghe Centre for Neurological Learning Disabilities (CNL) is an expertise centre for children with neurological learning disabilities such as NF1. At school age, a paediatric neurologist evaluates all patients at least once. Patients are offered additional evaluations by a neuropsychologist and a physiotherapist. Patients without any complaints about motor performance were not included in this study. Next to this, we did not reevaluate the motor performance of patients who already had serious motor problems according to a recent evaluation by a physiotherapist using the Movement-ABC in a different institute. The selection process is depicted in Fig. 1. We used medical and psychological patient files from 2002 to 2014 of 4- to 16-year-old patients who met the National Institutes of Health (NIH) diagnostic criteria for NF1 [12] and who were evaluated by a physiotherapist using the Movement Assessment Battery for Children version 1 [13] or 2 [14] (M-ABC-1 or 2). Exclusion criteria were segmental NF1, symptomatic pathology of the CNS, deafness or severely impaired vision, pseudarthrosis, insufficient command of the Dutch language, or an IQ below the range covered by the Wechsler Intelligence Scale for Children, third edition, Dutch version (WISC-III-NL [15]; total IQ below 48).

Clinical data were registered by a paediatrician of Erasmus Medical Centre, Sophia Children's Hospital during annual follow-up, by a paediatric neurologist from CNL and by psychologists from both centres. All children were evaluated according to a standardised protocol, routinely applied to all children with NF1 visiting the expertise centre. Familial or sporadic NF1 was determined from family history. In clinical assessments by the paediatric neurologist and the psychologist, the presence of neurologic, orthopaedic, or neuropsychiatric problems such as hypotonia, hypermobility, and scoliosis were recorded. Classifications of ADHD and ASD were based on neuropsychological assessment and on information from parents and teachers, using DSM-IV [16] criteria. Writing problems were reported by parents. Socio-

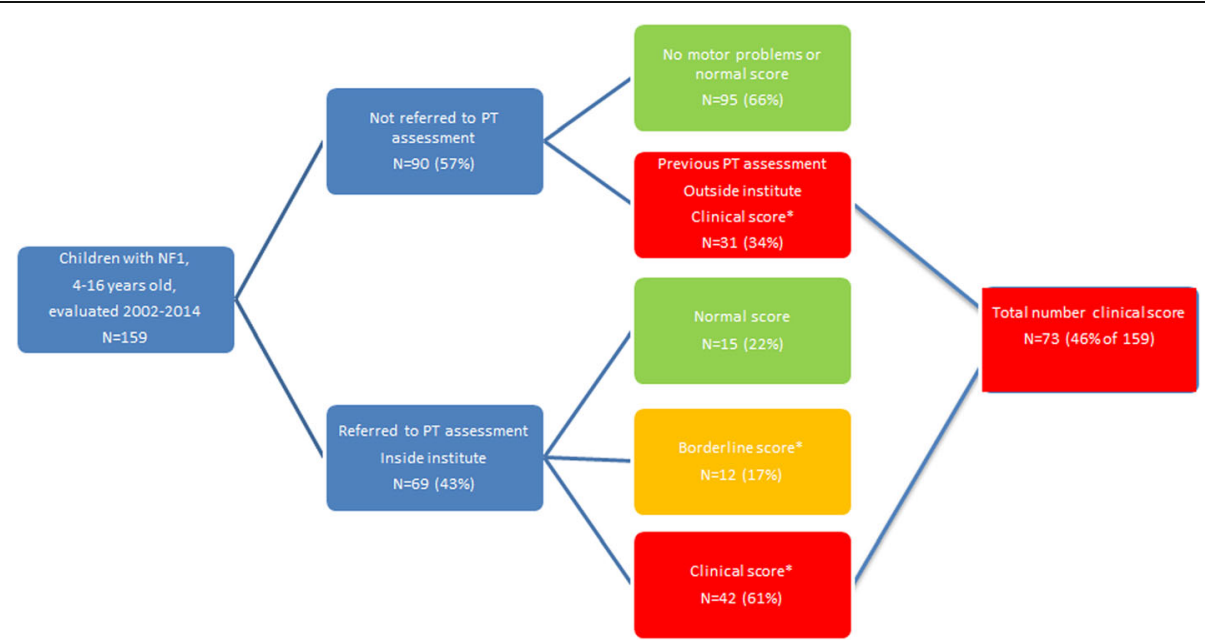

Fig. 1 Flow chart of participants and outcomes. * M-ABC normal score $>$ P15; borderline score P5 to <P15; clinical score $<P 5$ 
economic status (SES) was derived from the zip code of the child's home address using a standard Dutch classification system [17]. For the participating patients, a formal review and waiver was given by the medical ethical human research ethics committees of both the Erasmus Medical Centre and the CNL.

\section{Instruments}

The Movement $A B C$, $[13,14]$ an instrument measuring the presence and severity of motor problems, is one of the most frequently and widely used standardised assessments of motor skills, also used in diagnosing developmental coordination disorder (DCD). To assess motor performance, the physiotherapist administered the MABC-1 [13] (2002-2010) or 2 [14] (2010-2014). The M$\mathrm{ABC}$ assesses three components: manual dexterity, ball skills (catching and throwing), and balance (static and dynamic). The $\mathrm{M}-\mathrm{ABC}$ is designed to identify and describe impairments in the motor performance of children and adolescents aged 4 to 12 (M-ABC-1) or 3 to 16 $(\mathrm{M}-\mathrm{ABC}-2)$. The $\mathrm{M}-\mathrm{ABC}-2$ is an updated version of the $\mathrm{M}-\mathrm{ABC}-1$, not only the age range but also the sample size, have been expanded and more information on psychometric qualities has been acquired. Results on both tests are expressed in a score, with any child scoring below the 6th percentile of the normative sample being recorded as falling within the clinical range indicating serious movement difficulties. Scores from the $6^{\text {th }}$ to the $15^{\text {th }}$ percentile (approximately between 1.5 and $2.0 \mathrm{SD}$ below average) are labelled as borderline, indicating that the child is at risk of motor problems. Above the 15th percentile, the child is unlikely to have movement difficulties. Additionally, the $\mathrm{M}-\mathrm{ABC}-2$ also provides normreferenced standardised scores for the component and the total scores. The M-ABC-2 has good reliability (ICC $=.95$ to .98$)$.

Intelligence was measured with the Wechsler Preschool and Primary Scale of Intelligence-Revised, Dutch version (first WPPSI-R [18], from 2010 WPPSI-III-NL [19]) or the WISC-III-NL [15]. These are intelligence tests for children, the first for those aged 2 years and 6 months to 7 years and 7 months, and the second for children aged 6 to 17 years. The tests consist of several subtests resulting in a Full-Scale IQ, Verbal IQ, and Performance IQ.

To assess emotional and behavioural problems, parents completed the Child Behavior Checklist (CBCL) using either the preschool version, the $\mathrm{CBCL} / 1^{1 / 2}-5$ [20], or the school-aged version, CBCL/6-18 [21]. Scores were converted to $T$ scores (mean 50, SD 10), with higher scores corresponding to more problems. Summed scores result in three broadband scales for Internalising, Externalising, and Total Problems. The Internalising Problems scale comprises anxious/depressed behaviour, withdrawn/depressed behaviour, and somatic complaints. The Externalising Problems scale comprises rule-breaking behaviour and aggressive behaviour. The Total Problems scale is a combination of both the Internalising and Externalising Problems scales, together with scales for Social Problems, Thought Problems, and Attention Problems. $T$ scores between 59 and 62 fall within a borderline clinical range, whilst $T$ scores of 63 and higher fall within the clinical range. All tests were administered in their Dutch versions, using Dutch normative samples.

\section{Statistical analysis}

All data were analysed using SPSS, version 21 [22], and $R$ [23]. Proportions of groups were compared using chisquare $\left(\chi^{2}\right)$ tests. Effect sizes were calculated using Cohen's $d$, [24] when comparing the NF1 sample with the test manual normative sample, with .20 interpreted as a small effect size, .50 as medium, and .80 as large.

Since the common outcome for both versions of the $\mathrm{M}-\mathrm{ABC}$ was the classification into three consecutive categories (normal, borderline, or clinical), ordinal logistic regression analysis was performed to find predictors of these three categories of motor outcome. For this, a two-phase strategy was followed. In phase 1, all separate variables from Table 1 were tested in univariable ordinal regression analyses with $\mathrm{M}-\mathrm{ABC}$ classification as the dependent variable. Since this phase served as an initial, broad selection of potential predictors, $\alpha$ in phase 1 was set at .20 [25]. In phase 2 , multivariable ordinal regression models were constructed for every block of variables from Tables 1 and 2, containing all significant variables from phase 1 . Blocks were defined as demographics, neuropsychiatric problems, emotional and behavioural problems, and cognition. Variables shown to be significant contributors in the final models were regarded as the final predictors of $\mathrm{M}-\mathrm{ABC}$ motor outcome ( $\alpha$ in phase 2 was set at .05; stepwise backward elimination procedure).

\section{Results \\ Patient characteristics}

From 2002 to 2014, 159 children with NF1 aged 4 to 16 years old visited the expertise centre. Ninety (57\% of 159; 46 girls and 44 boys) were not referred to the physiotherapist, of which 31 (34\% of 90 ) had a previous assessment outside our institute, indicating serious motor problems, according to $\mathrm{M}-\mathrm{ABC}$ scores in the clinical range. Of the other 59 (66\% of 90$)$, parents and children did not have any complaints about motor performance before or during their visit to our institute. In the flow chart of Fig. 1, we have visualised the distribution of the sample. For this study, 69 children (43\% of 159) with NF1 were included for PT evaluation in our 
Table 1 Characteristics of children with NF1

\begin{tabular}{ll}
\hline Variable & \\
\hline Demographic characteristics & $n=69$ \\
Age & $8.7(4.1)^{\mathrm{a}}$ \\
Gender & \\
$\quad$ Male & $40(58)$ \\
$\quad$ Female & $29(42)$ \\
Type of education & \\
$\quad$ Regular education & $48(70)$ \\
$\quad$ Special education & $21(30)$ \\
$\quad$ Social economic status & \\
Mode of inheritance NF1 & $0.34(1.29)^{\mathrm{a}}$ \\
De novo mutation & \\
Familial mutation & $39(57)$ \\
Unknown & $29(42)$ \\
Neuropsychiatric problems & $1(1)$ \\
Attention-deficit/hyperactivity disorder (ADHD) & \\
ADHD combined type & \\
ADHD inattentive type & $25(36)$ \\
ADHD hyperactive/impulsive type & $11(16)$ \\
Total & $2(3)$ \\
Using stimulant medication & $38(55)$ \\
Heurologic and orthopaedic problems & $18(26)$ \\
\hline Hypotonia & $7(10)$ \\
\hline
\end{tabular}

${ }^{\text {a Median (interquartile range) }}$

${ }^{\mathrm{b}}$ Average SES in $2010=0.17$; higher scores indicate higher SES

institute, 29 girls, and 40 boys. This group is indicated in the box 'Referred to PT assessment' in Fig. 1. Ages ranged from 4 years to 15 years and 11 months, with a median age of 8 years and 8 months (IQR $=4$ years and 1 month) (Table 1). Sixty-seven children were right handed. Eighteen out of 38 of the children with a DSM-IV-TR classification of ADHD (47\%) used stimulant medication. Seven children had an ASD classification, all of them with a comorbid ADHD classification. Intelligence, emotional and behavioural problems, and standard scores of the MABC-2 are presented in Table 2.

Twenty-four children (41\%) had emotional and behavioural problem scores within the clinical range, with large effect sizes for internalising problems and medium effect sizes for externalising problems. Parents of 11 children (16\%) did not return CBCLs. These children were left out of analyses using CBCL scores as predictors. Compared to the normative sample, the distribution of intelligence scores was shifted approximately one
SD to the left, and total IQ scores ranged from 58 to 123. Effect sizes were large for performance IQ and medium for verbal IQ compared to the normative population. Effect sizes for all motor scales were large.

\section{Motor problems}

Thirty-five of 69 children (51\%) were assessed with the $\mathrm{M}-\mathrm{ABC}$ version 1,34 (49\%) with version 2 . The comparison between children tested with these two versions showed no significant differences in the distribution of scores between the percentile classification categories for the total scores $\left(\chi^{2}(2)=3.08, p=.21\right)$, nor for distributions of Hand, Ball, or Balance scale scores. For the purpose of ordinal regression analyses, both groups were combined. Figure 2 presents the distribution of the classifications in all motor scales. Overall, 42 (61\%) children with NF1 scored within the clinical range (below 6th percentile) of the M-ABC.

In ordinal regression analysis, age was found not to be a significant predictor of motor outcome. The proportion of children scoring in the 'borderline' or 'clinical' range of the $\mathrm{M}-\mathrm{ABC}$ was $67 \%$ of children from 4 to 6 years old, $82 \%$ of children from 7 to 11 years old, and $79 \%$ of adolescents from 12 to 16 years old.

In univariable ordinal regression analysis (Table 3; phase 1 with $\alpha$ set at .20), a higher probability of borderline or clinical motor problems was predicted by type of education, classifications of ADHD or ASD, hypermobility, Performance IQ, Total IQ, and CBCL Internalising, Externalising, and Total Problems. In all univariable models, the test of parallel lines failed to reach significance, meaning that effects of all separate variables were the same for normal versus borderline and borderline versus clinical scores.

In multivariable ordinal regression analyses (Table 4; phase 2 with $\alpha$ set at .05), single variables within one block (type of education and hypermobility) had $p$ values above $\alpha=.05$ and so could not be used in multivariable models. In three blocks, final models yielded a limited amount of significant predictors. Since all seven children with an ASD classification scored within the clinical range, the odds ratio of having borderline or clinical M-ABC scores could not be calculated and so ASD was left out of multivariable analyses. Also, the multivariable ordinal regression of ADHD and ASD could not be performed because all seven children with ASD classifications also had an ADHD classification. We compared the distribution of the $\mathrm{M}-\mathrm{ABC}$ classification between the groups without ADHD or ASD versus the group with only ADHD versus the group with both ADHD and ASD using a chi-squared test. This distribution did not differ significantly $\left(\chi^{2}(4, N=69)\right.$ $=7.53, p=.11)$, indicating that all three groups contributed independently to the distribution of motor problems. 
Table 2 Scores and frequencies for emotional and behavioural problems, intelligence and motor performance

\begin{tabular}{|c|c|c|c|c|c|c|}
\hline Domain & Number & Mean & $S D^{a}$ & $\mathrm{BCR}(\%)$ & $C R^{\mathrm{b}}(\%)$ & $\overline{E S^{c}}$ \\
\hline \multicolumn{7}{|c|}{ Parent-rated emotional and behavioural problems ${ }^{\mathrm{d}}$} \\
\hline Internalising problems & 58 & 59 & 10 & 19 & 37 & $0.9^{* * *}$ \\
\hline Externalising problems & 58 & 55 & 12 & 8 & 27 & $0.5^{* *}$ \\
\hline Total problems & 58 & 61 & 11 & 10 & 41 & $1.0^{* * *}$ \\
\hline \multicolumn{7}{|l|}{ Intelligence $e^{e}$} \\
\hline Verbal IQ & 68 & 92 & 15 & & & $0.5^{* * * *}$ \\
\hline Performance IQ & 68 & 88 & 14 & & & $0.8^{* * *}$ \\
\hline Total IQ & 69 & 89 & 13 & & & $0.8^{* * * *}$ \\
\hline \multicolumn{7}{|c|}{ Movement $\mathrm{ABC}-1$ and $2(n=69)$} \\
\hline Classification normalf & $15(22 \%)$ & & & & & \\
\hline Classification borderline $e^{f}$ & $12(17 \%)$ & & & & & \\
\hline Classification clinical $^{f}$ & $42(61 \%)$ & & & & & \\
\hline \multicolumn{7}{|l|}{ Movement $\mathrm{ABC}-2^{\mathrm{g}}(n=34)$} \\
\hline Manual dexterity & 34 & 5.8 & 3.3 & & & $1.3^{* * * *}$ \\
\hline Ball skills & 34 & 6.7 & 3.6 & & & $1.0^{* * *}$ \\
\hline Balance & 33 & 5.7 & 3.0 & & & $1.4^{* * *}$ \\
\hline Total & 34 & 4.8 & 3.2 & & & $1.7^{* * *}$ \\
\hline
\end{tabular}

${ }^{a} S D$ Standard deviation

${ }^{\mathrm{b}} B C R / C R$ Percentage of scores in borderline clinical range/clinical range

'ES effect size (Cohen's $d$ ); Significance compared to normative sample ${ }^{* *} p<.01 ;{ }^{* * * *} p<.001$

${ }^{\mathrm{d}} T$ scores (population mean $=50 ; \mathrm{SD}=10$; higher scores reflect more problems)

${ }^{\mathrm{e}} \mathrm{IQ}$ scores (population mean $=100 ; \mathrm{SD}=15$; higher scores reflect better performance)

${ }^{f} M-A B C$ normal score $>$ P15; borderline score P5 to $<$ P15; clinical score $<P 5$ )

${ }^{9}$ Standard-scores (population mean $=10 ; \mathrm{SD}=3$; higher scores reflect better performance)

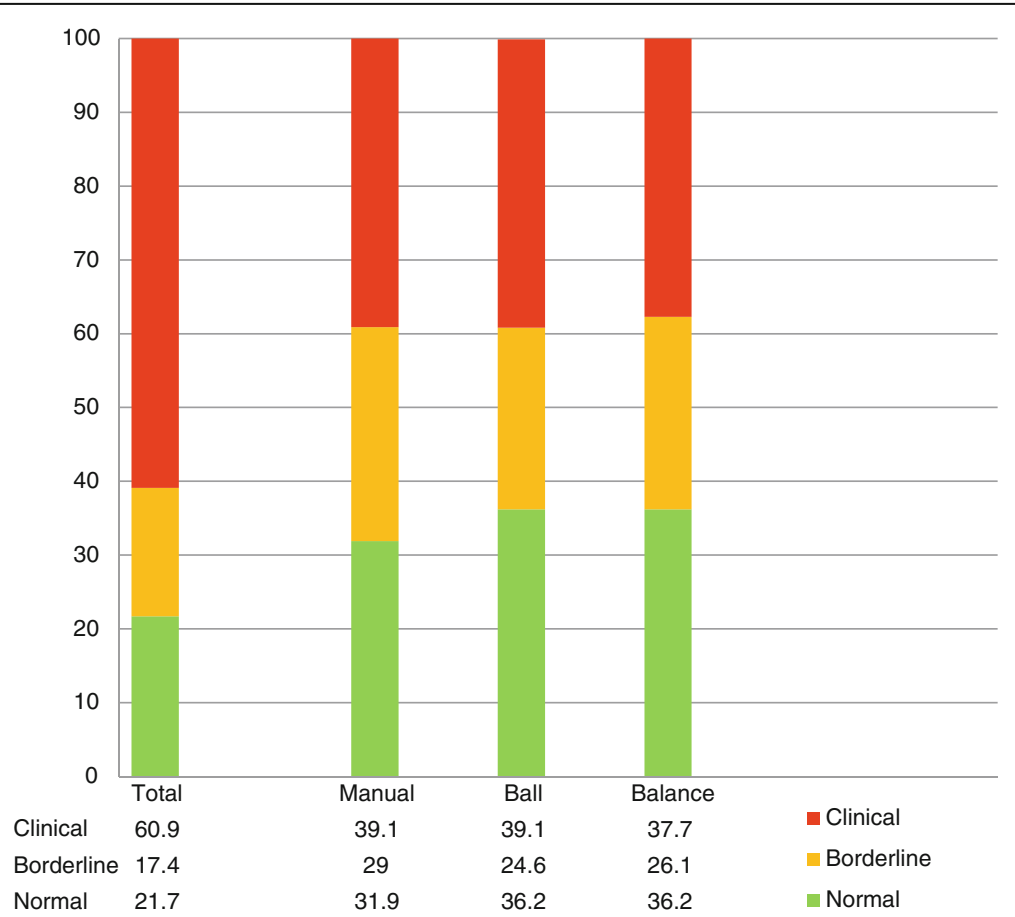

Fig. 2 Classification of motor problems based on Movement ABC percentile scores $(n=69)$. Clinical: percentage of children with movement difficulty- scores below 6th percentile. Borderline: percentage of children with scores from 6th to 15th percentile. Normal: percentage of children with no movement difficulty scores above 15 th percentile 
Table 3 Univariable ordinal logistic regression with separate variables predicting motor outcome (Movement ABC total $s c o r e s ; n=69$ )

\begin{tabular}{|c|c|c|c|c|c|c|c|c|}
\hline \multirow[t]{2}{*}{ Variable } & \multirow[t]{2}{*}{ Number } & \multirow[t]{2}{*}{$\mathrm{B}(\mathrm{SE})$} & \multicolumn{3}{|c|}{$95 \% \mathrm{Cl}$ of odds ratio } & \multirow[t]{2}{*}{ Wald } & \multirow[t]{2}{*}{$R^{2}$} & \multirow[t]{2}{*}{$p$} \\
\hline & & & Lower & OR & Upper & & & \\
\hline Age & 69 & $0.08(0.09)$ & 0.77 & 0.93 & 1.12 & 4.31 & .01 & .429 \\
\hline Gender & 69 & $0.56(0.49)$ & 0.22 & 0.57 & 1.49 & 1.31 & .02 & .253 \\
\hline Type of education & 69 & $0.84(0.58)$ & 0.14 & 0.43 & 1.35 & 2.08 & .04 & $.135^{\#}$ \\
\hline Social economic status & 69 & $-0.10(0.19)$ & 0.77 & 1.10 & 1.59 & 0.29 & .01 & .595 \\
\hline Mode of inheritance & 69 & $-0.42(0.49)$ & 0.59 & 1.53 & 3.95 & 0.76 & .01 & .383 \\
\hline $\mathrm{ADHD}$ & 69 & $1.01(0.49)$ & 0.14 & 0.36 & 0.96 & 4.22 & .07 & $.038^{*}$ \\
\hline Using stimulant medication & 69 & $-0.35(0.54)$ & 0.49 & 1.41 & 4.05 & 0.41 & .01 & .523 \\
\hline Autism spectrum disorder ${ }^{a}$ & 69 & - & - & NA & - & - & .12 & $.035^{*}$ \\
\hline Hypotonia & 69 & $0.28(0.60)$ & 0.23 & 0.76 & 2.47 & 0.21 & $<.01$ & .644 \\
\hline Hypermobility & 69 & $0.98(0.70)$ & 0.10 & 0.38 & 1.49 & 1.94 & .04 & $.140^{\#}$ \\
\hline Scoliosis & 69 & $0.25(0.79)$ & 0.28 & 1.28 & 5.97 & 0.10 & $<.01$ & .755 \\
\hline Writing problems at school & 69 & $-0.20(0.50)$ & 0.46 & 1.22 & 3.25 & 0.16 & $<.01$ & .694 \\
\hline CBCL Internalising problems & 58 & $-0.04(0.03)$ & 0.99 & 1.04 & 1.09 & 2.23 & .04 & $.134^{\#}$ \\
\hline CBCL Externalising problems & 58 & $-0.04(0.02)$ & 0.10 & 1.04 & 1.09 & 3.30 & .07 & $.063^{\#}$ \\
\hline $\mathrm{CBCL}$ Total problems & 58 & $-0.05(0.03)$ & 1.00 & 1.05 & 1.10 & 3.65 & .08 & $.051^{\#}$ \\
\hline Verbal IQ & 68 & $0.01(0.02)$ & 0.96 & 0.99 & 1.02 & 0.41 & .01 & .519 \\
\hline Performance IQ & 68 & $0.03(0.02)$ & 0.94 & 0.97 & 1.01 & 2.43 & .04 & $.115^{\#}$ \\
\hline Total IQ & 69 & $0.03(0.02)$ & 0.94 & 0.97 & 1.01 & 2.10 & .04 & $.141^{\#}$ \\
\hline
\end{tabular}

$R^{2}$ Nagelkerke pseudo $R^{2}$

$p$ values of likelihood ratio chi-square; ${ }^{*} p<.20 ;{ }^{*} p<.05 ;{ }^{* *} p<.01$

${ }^{a}$ As there were no cases in cells with normal M-ABC-scores for children with an ASD classification, the estimate was minus infinity

Table 4 Multivariable backward ordinal logistic regression with variables from separate blocks predicting motor outcome (Movement ABC total classification; $n=69$ )

\begin{tabular}{|c|c|c|c|c|c|c|c|c|}
\hline \multirow[t]{2}{*}{ Variables } & \multirow[t]{2}{*}{ Number } & \multirow[t]{2}{*}{$\mathrm{B}(\mathrm{SE})$} & \multicolumn{3}{|c|}{$95 \% \mathrm{Cl}$ of $\mathrm{OR}$} & \multirow[t]{2}{*}{ Wald } & \multirow[t]{2}{*}{$R^{2}$} & \multirow{2}{*}{$\begin{array}{l}p \\
\text { value }\end{array}$} \\
\hline & & & Lower & OR & Upper & & & \\
\hline \multicolumn{9}{|l|}{ Neuropsychiatric problems } \\
\hline ADHD & 69 & $1.01(0.49)$ & 0.14 & 0.36 & 0.96 & 4.22 & .07 & $.038^{*}$ \\
\hline Autism spectrum disorder ${ }^{a}$ & 69 & - & - & NA & - & - & .12 & $.035^{*}$ \\
\hline \multicolumn{9}{|c|}{ Emotional and behavioural problems } \\
\hline Model 1 & 58 & & & & & & .07 & .168 \\
\hline Internalising problems & & $-0.01(0.03)$ & 0.95 & 1.01 & 1.08 & 0.10 & & .757 \\
\hline Externalising problems & & $-0.04(0.03)$ & 0.98 & 1.04 & 1.10 & 1.44 & & .235 \\
\hline Model 2 & 58 & & & & & & & \\
\hline Externalising problems & & $-0.04(0.02)$ & 0.10 & 1.04 & 1.09 & 3.30 & .07 & .063 \\
\hline \multicolumn{9}{|l|}{ Intelligence } \\
\hline Model 1 & 68 & & & & & & .04 & .289 \\
\hline Performance IQ & & $0.03(0.03)$ & 0.91 & 0.97 & 1.04 & 0.71 & & .401 \\
\hline Total IQ & & $0.001(0.03)$ & 0.94 & 1.00 & 1.07 & 0.001 & & .973 \\
\hline Model 2 & 68 & & & & & & & \\
\hline Performance IQ & & $0.03(0.02)$ & 0.94 & 0.97 & 1.01 & 2.43 & .04 & .115 \\
\hline
\end{tabular}

OR odds ratio, NA not applicable, $R^{2}$ Nagelkerke pseudo $R^{2}$

$p$ values of likelihood ratio chi-square; ${ }^{*} p<.05$

${ }^{a}$ As there were nog cases in cells with normal M-ABC-scores for children with an ASD classification, the estimate was minus infinity 
The Externalising Problems scale was approaching significance as a predictor of motor outcome $(p=.063)$. With low scores for Externalising Problems, the probability of a clinical score on the M-ABC was low. Children without externalising problems on the CBCL only had a $23 \%$ chance of a clinical score on the M-ABC, whilst children with externalising problems scores in the clinical range had an $81 \%$ chance, as is shown in Fig. 3. Finally, intelligence (i.e. Performance IQ) was not found to be significantly associated with total motor problems.

Exploratory univariable linear regression analyses, with motor outcome on the $\mathrm{M}-\mathrm{ABC}-2$ as a continuous dependent variable $(n=34)$, found significant associations with independent variables: Internalising Problems scale $\left(F(1,26)=5.21 ; p=.031 ; R^{2}=.17 ; \beta=-.13\right)$; Externalising Problems scale $\left(F(1,26)=6.99 ; p=.014 ; R^{2}\right.$ $=.21 ; \beta=-.12)$; and Total Problems scale $(F(1,26)=6.15$; $\left.p=.020 ; R^{2}=.19 ; \beta=-.13\right)$, again indicating that an increase in emotional and behavioural problems is associated with a decrease in motor proficiency. Residuals for these regressions were normally distributed.

\section{Discussion}

Our study shows that motor problems frequently occur in our group of children with NF1: $61 \%$ of these 69 children have serious motor problems and another $17 \%$ score within the borderline range. In the part of our cohort not evaluated in the expertise centre $(n=90), 31$ were already identified as having motor problems, resulting in an overall $46 \%(73 / 159)$ with serious motor problems. The distribution of these groups and outcomes is visualised in Fig. 1 in the box with 'Total number clinical

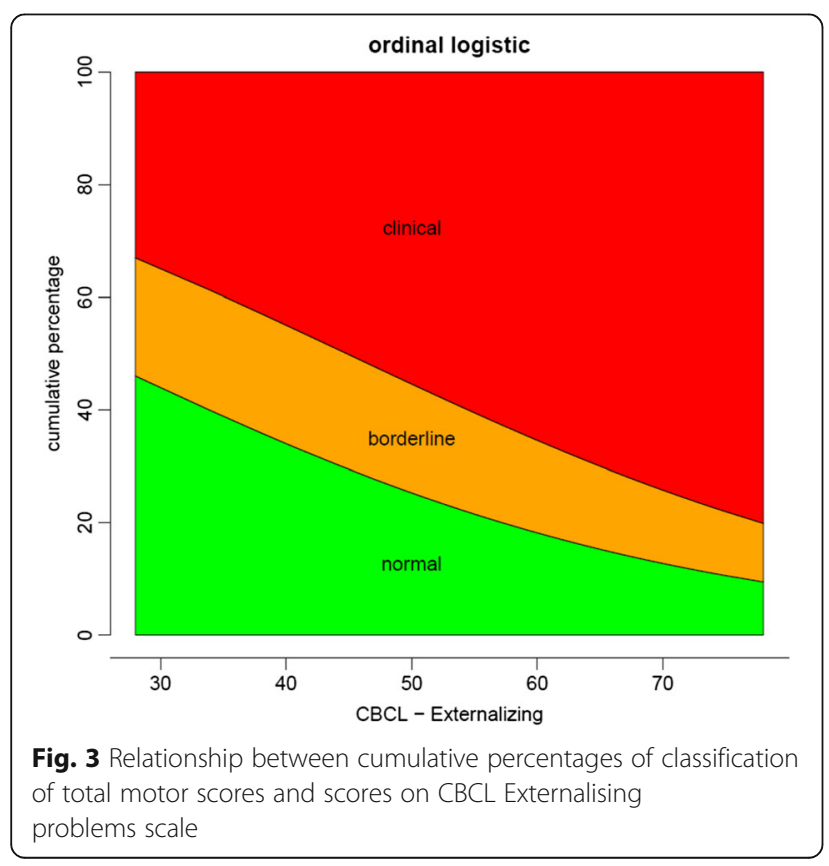

score'. Previous studies using broad motor test batteries found smaller or comparable proportions. One study in a comparable age range found $54 \%$ (14 out of 26 children) scoring between one and two standard deviations below average and another 27\% (7/26) scored below 2 SD [6]. When comparing studies, one should realise that the cut-offs of the P5 and the P15 correspond to $z$ scores of 1.65 and 1.04 below average in the standard normal distribution.

Next to ADHD [26] and ASD symptoms [27], motor problems seem to be among the most common comorbid developmental problems of children with NF1. We found motor problems in a broad range of domains, comparable to the problems found in DCD [8].

In our attempt to find predictors of motor outcome, we did not find a significant contribution of demographic characteristics such as age, gender, or SES. A previous comparable study in a smaller sample did not find effects for age or gender either [6]. We also did not find associations with neurological and orthopaedic problems such as hypotonia, hypermobility, or scoliosis. Given the broad variability in these characteristics within our population (Table 1), we think our study population had sufficient power to detect potential associations if they existed. There was a limited association between (performance) intelligence and motor performance. Previous research [4] found that motor coordination and motor speed contributed to the performance on some subtests of the WISC. However, in our study, we used a broader motor test battery such as the M-ABC and children were found to have serious motor problems in general, regardless of their overall intelligence. Since a previous study [9] found that poorer balance skills were associated with a reduced perceptual reasoning index, we performed an additional univariable ordinal logistic regression to specifically find out whether balance skills on the M-ABC were associated with performance IQ. Only a weak association was found with an odds ratio of 0.97 (95\% CI, 0.93 to 1.00 ), Wald $\chi^{2}(1)=3.774, p=.052$ ). Whether this finding is a reflection of an abnormal neurodevelopmental process, underlying these abilities in children with NF1, may be a subject for future research.

Recent studies do provide evidence for a relation between motor experience and cognitive development in the first 3 years of life when at the same time this relation becomes less clear in older children [28]. The fact that we did not find a significant effect of age on motor performance may presumably be caused by the fact we included children from 4 to 16 years old.

Externalising behavioural problems might be associated with motor outcome. This association was found to be significant in additional explorative analyses with standard scores of the children tested with the M-ABC2. Also, ADHD was a significant predictor of motor 
outcome, and all children with an ASD classification had severe motor problems. Previous studies also found that motor problems often occur in children with emotional, behavioural, and pervasive developmental disorders [29, 30]. The co-occurrence of motor and behavioural problems could be an indicator of a more severe neurologic phenotype [31]. It is, however, unclear what the direction of the association between behavioural and motor problems is. Longitudinal and treatment studies could elucidate this issue. Neuropsychiatric and motor problems have a large impact on participation in daily life, even more so when these problems occur simultaneously.

\section{Limitations}

Although NF1 is relatively rare, we succeeded in gathering data on the motor performance of 69 children over a 12 -year period. However, our sample size is still small considering the number of variables incorporated into the regression analyses of this study. For this reason, there is a risk of overfitting, and care should be taken when drawing conclusions regarding the predictive value of variables. To avoid unnecessary assessments, we did not evaluate the motor performance of children who recently had such an assessment. In addition, since the assessment of motor performance was on a voluntary basis, children without any motor complaints were not required to visit our physiotherapist. For these reasons, we cannot exclude selection bias. We tried to correct for this bias by calculating the total amount of children scoring in the clinical range (Fig. 1).

The cross-sectional design limits interpretations regarding the effect of age on motor performance. Probably, longitudinal research will be able to express this relationship in a more decisive way.

During the time period of this study, there was a move by physiotherapists in the Netherlands from using the first version of the Movement-ABC to the second version. For this reason, we were dependent on the categorical classification of motor problems as a primary outcome measure. This is a consequence of continuous sampling over a long period of time. One should be careful when combining data from both tests since the $\mathrm{M}-\mathrm{ABC}-2$ is an elaboration of the $\mathrm{M}-\mathrm{ABC}-1$, resulting in differences between both instruments [32]. Because the age range of the $\mathrm{M}-\mathrm{ABC}$ was the starting point of this study, we used the two age-appropriate versions of the Wechsler scales and of the CBCL. Although the correlation between both versions is high, $[19,20]$ future research in larger groups could benefit from the selection of smaller age ranges.

For this study, we collected data from medical records. This resulted in missing information (as is shown in Table 2), particularly regarding emotional and behavioural problems, most likely because some parents failed to return questionnaires. Since all children were assessed using a standardised protocol, other data are relatively complete.

The proportion of children with ADHD symptoms is comparable to that in other studies, [26] but the percentage of children with ASD symptomatology in our study (10\%) is somewhat lower than former prevalence estimates (21-40\%) [33]. In the group with ASD, all children appeared to have severe motor problems. Although this may suggest clinical relevance, we interpret this observation with care, due to the small sample size.

\section{Clinical implications and recommendations}

Developmental motor problems are frequently overlooked in clinical practice, yet they can have a considerable impact on children's lives [34]. Using a broad motor assessment in a large cohort of children with NF1, we showed a high prevalence of serious motor problems. These problems seem to be independent of age or intelligence. When children with NF1 show serious motor problems, the diagnosis of DCD might be considered as a comorbid problem. This is especially important in helping to recognise the impact of motor problems on daily life and in allocating the correct treatment. Although the DSM-IV-TR [16] states that in DCD, 'the disorder is not due to a general medical condition', to our opinion NF1 does not have to be regarded as such. DCD could be used in practice as a descriptive diagnosis stressing the impact of motor problems on daily life.

Concerning participation in daily life, children with NF1 often experience problems with writing $[4,35]$. It is important to find out whether people with NF1 experience further such difficulties in daily functioning such as in activities of daily living, play, sports, or with driving. This is of great importance since a decrease in participation could not only affect the practice of motor skills but also the development of social skills and quality of life in general.

Assessment and treatment of motor problems in NF1, especially in children with behavioural and social problems, should be considered at a young age, using a broad motor assessment battery. Early motor intervention can have a beneficial effect on behavioural problems, as is indicated by a study showing that in ADHD, [36] motoraffected children receiving physiotherapy presented less frequently with comorbid emotional and behavioural problems. The impact of physiotherapy and psychological therapy on motor functioning, motor participation, and emotional and behavioural problems in children with both NF1 and motor problems is unknown. However, considering the larger potential for plasticity at a young age, referral to both a physiotherapist and a psychologist could be considered at a young age in children with NF1. 


\section{Conclusions}

More than half of the children with NF1 in this sample had severe motor problems. These problems seem to be independent of age or intelligence. Next to ADHD and ASD, motor problems are among the most frequent comorbid developmental problems in children with NF1. In this study, ADHD and ASD symptomatology, and externalising behavioural problems are associated with motor problems. The combination of both motor and behavioural problems might result in a more severe phenotype of NF1. Because of their impact on participation in daily life, motor problems need to be specifically addressed in diagnosis, follow-up, and treatment of children with NF1.

\section{Abbreviations \\ ADHD: Attention-deficit/hyperactivity disorder; ASD: Autism spectrum disorder; CBCL: Child Behavior Checklist; CNL: Kempenhaeghe Centre for Neurological Learning Disabilities; DCD: Developmental coordination disorder; DSM-IV: Diagnostic and statistical manual of mental disorders; IQ: Intelligence quotient; M-ABC-1 or 2: Movement Assessment Battery for Children version 1 or 2; NF1: Neurofibromatosis type 1; SES: Socio-economic status; WISC-III-NL: Wechsler Intelligence Scale for Children, third version for the Netherlands; WPPSI-III-R: Wechsler Preschool and Primary Scale of Intelligence-Third version for the Netherlands}

\section{Acknowledgements}

We would like to thank all the children and families for participating in this study. A special thank you to Bethany Nicholson for her assistance and expertise, and Alma Weber and Annick Laridon for including many patients and for the assistance during study conduct. We also thank clinicians throughout the Netherlands for referring patients.

\section{Funding}

This study was not supported by any grant.

\section{Availability of data and materials}

The datasets used and/or analysed during the current study are available from the corresponding author on reasonable request.

\section{Authors' contributions}

ABR planned and conceptualised the study, drafted the initial manuscript, performed all of the statistical analyses, created all of the tables, and edited the final manuscript as submitted. SB participated in the planning of the study, participated in drafting the initial manuscript, and assisted with statistical analyses. EG, SvA, JGH, and PFAdN participated in the interpretation of the results and edited the final manuscript as submitted. CWNL assisted with statistical analyses and edited the final manuscript as submitted. RO and M-CdW supervised the study design and the analysis, participated in the interpretation of the results, and edited the final manuscript as submitted. All authors approved the final manuscript as submitted.

\section{Competing interests}

The authors declare that they have no competing interests.

\section{Consent for publication}

Not applicable.

\section{Ethics approval and consent to participate}

For the participating patients, a formal review and waiver were given by the medical ethical human research ethics committees of both the Erasmus Medical Centre and the CNL. Reference number: MEC-2015-203.

\section{Publisher's Note}

Springer Nature remains neutral with regard to jurisdictional claims in published maps and institutional affiliations.

\section{Author details}

${ }^{1}$ Department of Child and Adolescent Psychiatry/Psychology, ENCORE NF1 Expertise Centre for Neurodevelopmental Disorders, Erasmus Medical Centre-Sophia Children's Hospital, Rotterdam, The Netherlands. ${ }^{2}$ Department of General Paediatrics, ENCORE NF1, Erasmus Medical Centre-Sophia Children's Hospital, Rotterdam, The Netherlands. ${ }^{3}$ Department of Paediatric Neurology, ENCORE NF1, Erasmus Medical Centre-Sophia Children's Hospital, Rotterdam, The Netherlands. ${ }^{4}$ Department of Public Health, Erasmus Medical Centre, Rotterdam, The Netherlands. ${ }^{5}$ Kempenhaeghe Centre for neurological learning disabilities, Heeze, The Netherlands. ${ }^{6}$ Department of Child and Adolescent Psychiatry/Psychology, Sophia Children's Hospital, Room Sp 2478, P.O. Box 20603000 CB Rotterdam, The Netherlands.

Received: 20 September 2016 Accepted: 2 May 2017

Published online: 19 May 2017

\section{References}

1. Evans DG, Howard E, Giblin C, Clancy T, Spencer H, Huson SM, et al. Birth incidence and prevalence of tumor-prone syndromes: estimates from a UK family genetic register service. Am J Med Genet A. 2010;152A(2):327-32. doi: 10.1002/ajmg.a.33139.

2. Lehtonen A, Howie E, Trump D, Huson SM. Behaviour in children with neurofibromatosis type 1: cognition, executive function, attention, emotion, and social competence. Dev Med Child Neurol. 2013;55(2):111-25. doi:10. 1111/j.1469-8749.2012.04399.x.

3. Garg S, Lehtonen A, Huson SM, Emsley R, Trump D, Evans DG, et al. Autism and other psychiatric comorbidity in neurofibromatosis type 1: evidence from a population-based study. Dev Med Child Neurol. 2013;55(2):139-45. doi:10.1111/dmcn.12043

4. Soucy EA, Gao F, Gutmann DH, Dunn CM. Developmental delays in children with neurofibromatosis type 1. J Child Neurol. 2012;27(5):641-4. doi:10.1177/ 0883073811423974.

5. Johnson BA, MacWilliams BA, Carey JC, Viskochil DH, D'Astous JL, Stevenson DA. Motor proficiency in children with neurofibromatosis type 1. Pediatr Phys Ther. 2010;22(4):344-8. doi:10.1097/PEP.0b013e3181f9dbc8.

6. Hyman SL, Shores A, North KN. The nature and frequency of cognitive deficits in children with neurofibromatosis type 1. Neurology. 2005;65(7): 1037-44. doi:10.1212/01.wnl.0000179303.72345.ce.

7. Krab LC, Aarsen FK, de Goede-Bolder A, Catsman-Berrevoets CE, Arts WF, Moll HA, et al. Impact of neurofibromatosis type 1 on school performance. J Child Neurol. 2008;23(9):1002-10. doi:10.1177/0883073808316366.

8. Skinner RA, Piek JP. Psychosocial implications of poor motor coordination in children and adolescents. Hum Mov Sci. 2001;20(1-2):73-94.

9. Champion JA, Rose KJ, Payne JM, Burns J, North KN. Relationship between cognitive dysfunction, gait, and motor impairment in children and adolescents with neurofibromatosis type 1. Dev Med Child Neurol. 2014 56(5):468-74. doi:10.1111/dmcn.12361.

10. Hofman KJ, Harris EL, Bryan RN, Denckla MB. Neurofibromatosis type 1: the cognitive phenotype. J Pediatr. 1994;124(4):S1-8.

11. Lorenzo J, Barton B, Acosta MT, North K. Mental, motor, and language development of toddlers with neurofibromatosis type 1. J Pediatr. 2011; 158(4):660-5. doi:10.1016/j.jpeds.2010.10.001.

12. NIH nioh. Neurofibromatosis. NIH Consens Statement 1987 Jul 13-15. 1987; 6(12):1-19.

13. Henderson SE, Sugden DA. Movement assessment battery for children manual. London: The Psychological Corporation Ltd.; 1992.

14. Henderson S, Sugden D, Barnett A. Movement assessment battery for children-2 second edition [Movement ABC-2]. London: The Psychological Corporation; 2007

15. Kort W, Schittekatte M, Dekker PH, Verhaeghe P, Compaan EL, Bosmans M et al. WISC-III NL; Wechsler intelligence scale for children, derde Editie NL. Handleiding en verantwoording Londen: Harcourt Assessment; 2005.

16. Association AP. Diagnostic and statistical manual of mental disorders: DSMIV-TR. Washington, DC: American Psychiatric Association; 2000.

17. Knol FA. Statusontwikkeling van wijken in Nederland 1998-2010 (Dutch; 'Status development in districts in the Netherlands 1998-2010'). The Hague, the Netherlands: Social and Cultural Planbureau, 2012.

18. G. van der Steene AB. Wechsler Preschool and Primary Scale of Intelligence- Revised, Dutch version (WPPSI-R), manual. Lisse: Swets and Zeitlinger BV; 1997. 
19. Weschler D, Hendriksen J, Hurks P. WPPSI-III-NL, Wechsler preschool and primary scale of intelligence. Nederlandse bewerking. London: Pearson Assessment and Information BV; 2009.

20. Achenbach T, Rescorla LA. Multicultural Supplement to the Manual for the ASEBA Preschool Forms \& Profiles. Burlington: University of Vermont, Research Center for Children, Youth and Families; 2010.

21. Verhulst F, Van der Ende, J. Handleiding ASEBA: Vragenlijsten voor Leeftijden 6 t/m 18 jaar. Rotterdam: ASEBA Nederland; 2013.

22. IBMCorp. IBM SPSS Statistics for Windows, Version 20.0. Armonk, NY: IBM Corp.; 2011.

23. Team RDC. R: A language and environment for statistical computing. Vienna, Austria: R Foundation for Statistical Computing; 2008.

24. Cohen J. Statistical power analysis for the behavioral sciences. New York: Academic Press, New York; 1988.

25. Steyerberg E. Clinical prediction models: a practical approach to development, validation, and updating. New York: Springer; 2009.

26. Mautner VF, Kluwe L, Thakker SD, Leark RA. Treatment of ADHD in neurofibromatosis type 1. Dev Med Child Neurol. 2002;44(3):164-70.

27. Garg S, Plasschaert E, Descheemaeker MJ, Huson S, Borghgraef M, Vogels A, et al. Autism spectrum disorder profile in neurofibromatosis type I. J Autism Dev Disord. 2015;45(6):1649-57. doi:10.1007/s10803-014-2321-5.

28. Libertus K, Hauf P. Editorial: motor skills and their foundational role for perceptual, social, and cognitive development. Front Psychol. 2017;8:301. doi:10.3389/fpsyg.2017.00301.

29. Emck C, Bosscher R, Beek P, Doreleijers T. Gross motor performance and self-perceived motor competence in children with emotional, behavioural, and pervasive developmental disorders: a review. Dev Med Child Neurol. 2009:51(7):501-17. doi:10.1111/j.1469-8749.2009.03337.x.

30. Fliers E, Rommelse N, Vermeulen SH, Altink M, Buschgens CJ, Faraone SV, et al. Motor coordination problems in children and adolescents with ADHD rated by parents and teachers: effects of age and gender. J Neural Transm (Vienna). 2008;115(2):211-20. doi:10.1007/s00702-007-0827-0.

31. Peters $L H$, Maathuis $C G$, Hadders-Algra M. Children with behavioral problems and motor problems have a worse neurological condition than children with behavioral problems only. Early Hum Dev. 2014;90(12):803-7. doi:10.1016/j.earlhumdev.2014.09.001.

32. Brown T, Lalor A. The movement assessment battery for children — second edition (MABC-2): a review and critique. Phys Occup Ther Pediatr. 2009; 29(1):86-103. doi:10.1080/01942630802574908.

33. Garg S, Plasschaert E, Descheemaeker MJ, Huson S, Borghgraef M, Vogels A et al. Autism spectrum disorder profile in neurofibromatosis type I. J Autism Dev Disord. 2014. doi:10.1007/s10803-014-2321-5.

34. Baird G, Santosh PJ. Interface between neurology and psychiatry in childhood. J Neurol Neurosurg Psychiatry. 2003;74 Suppl 1:117-22.

35. Gillboa Y, Josman N, Fattal-Valevski A, Toledano-Alhadef H, Rosenblum S. The handwriting performance of children with NF1. Res Dev Disabil. 2010; 31(4):929-35. doi:10.1016/j.ridd.2010.03.005.

36. Fliers EA, Franke B, Lambregts-Rommelse NN, Altink ME, Buschgens CJ, der Nijhuis-van Sanden MW, et al. Undertreatment of motor problems in children with ADHD. Child Adolesc Ment Health. 2009;15(2):85-90. doi:10. 1111/j.1475-3588.2009.00538.x.

\section{Submit your next manuscript to BioMed Central and we will help you at every step:}

- We accept pre-submission inquiries

- Our selector tool helps you to find the most relevant journal

- We provide round the clock customer support

- Convenient online submission

- Thorough peer review

- Inclusion in PubMed and all major indexing services

- Maximum visibility for your research

Submit your manuscript at www.biomedcentral.com/submit
Biomed Central 